\title{
Breast Asymmetry Evaluation Using Objective Measures after Breast Cancer Surgery
}

\author{
Jaejong Park ${ }^{1}$, Fariha M. Haque ${ }^{2}$, Achille Louodom Chedjou1 ${ }^{1}$, Michael J. Miller ${ }^{3}$, Alok Sutradhar ${ }^{2}$ \\ ${ }^{1}$ Department of Mechanical Engineering, Prairie View A\&M University, Prairie View, TX, USA; ${ }^{2}$ Department of \\ Mechanical and Aerospace Engineering, The Ohio State University, Columbus, OH, USA; ${ }^{3}$ Plastic and \\ Reconstructive Surgery, Banner MD Anderson Cancer Center, Gilbert, AZ, USA
}

Correspondence to: Alok Sutradhar, sutradhar.1@osu.edu

Keywords: Asymmetry Breast Surgery, Outcome Research, Surface Imaging, 3D Scan

Received: March 31, $2020 \quad$ Accepted: January 23, $2021 \quad$ Published: January 26, 2021

Copyright $\odot 2021$ by author(s) and Scientific Research Publishing Inc.

This work is licensed under the Creative Commons Attribution-NonCommercial International License (CC BY-NC 4.0).

http://creativecommons.org/licenses/by-nc/4.0/

\section{(c) (1) $(9)$ Open Access}

\section{ABSTRACT}

Although a positive cosmetic outcome is an important goal of breast cancer reconstruction, the objective analysis of breast aesthetics has yet to reach a gold standard or unified method to quantify breast symmetry. Several scoring systems, both subjective and objective, have been developed over the years to ensure the desirable outcome in breast symmetry, but these methods have yet to reach the unanimous acceptance in terms of accuracy, value and ease of use to be implemented in the clinical setting. By assessing several existing symmetry scores, most of which are based on 2D imaging, along with our own set of symmetry parameters applied to $3 \mathrm{D}$ patient images, the goal of this study is to determine if there is an advantage of $3 \mathrm{D}$ imaging in formulating an accurate objective breast aesthetic score over the existing objective scores. A reliable breast aesthetic score would improve the decision-making in surgery as well as improve patient satisfaction. Additionally, knowing the quantity and degree of breast asymmetry objectively will improve outcome and reduce revision rates, minimizing patient suffering and improving the overall quality of patient life and body image.

\section{INTRODUCTION}

With the goal to restore the sense of wholeness and femininity as well as to erase the constant physical reminder of their battle with breast cancer, a large number of post mastectomy patients choose to undergo reconstructive breast surgery, either by TRAM (transverse rectus abdominis) flap or expander/ implant procedures to repair the breast deformities. Patient satisfaction with breast reconstructive surgery, whether be it breast cancer conservation therapy or aesthetic alteration, affects the quality of life and self-esteem of the patient [1]. While patients frequently indicate positive satisfaction with breast re- 
construction-either with breast cancer conservation therapy or aesthetic alteration-there are many cases where the patients are dissatisfied with the alterations that render the final aesthetic appearance of the surgical procedure [2].

There are certain aspects that influence patient satisfaction with the cosmesis. The dominant parameters in determining the overall breast symmetry include volume of the breast, shape and size, skin changes and scarring and the contour, as well as the position of the breasts. In order to determine the breast symmetry, subjective evaluation based on visual assessment is widely used. In the past, breast surgery outcome was analyzed subjectively by one or more observers, including radiation oncologists, plastic surgeons and even the patients themselves. Pusic et al. [3] proposed a conceptual framework (Breast-Q) using a list of questionnaires to measure surgery outcomes based on patient responses. It has shown its effectiveness in quantifying post-surgery satisfaction from the patients' perspective. However, subjective analyses has also been reported to be poorly reproducible and fail to adequately capture the degree of aesthetic difference between the breasts $[4,5]$. In order to reduce this inter-observer variability, multiple reviewers with differing backgrounds created scoring scales [4, 6-9].

A number of objective methods based on landmark measurements or volume measurements have been developed over the years. Pezner et al. $[9,10]$ initiated an objective assessment measure of breast aesthetic results by using an objective parameter: the breast retraction index which measures nipple location, based on the location of the mid-clavicle and sternal notch, and compares both sides to obtain a difference value. Though this index compares only nipple symmetry, a good correlation was demonstrated with subjective votes of breast symmetry. Van Limbergen et al. [11] furthered the use of nipple retraction in assessing breast shape by additionally measuring the distance to the breast borders. Noguchi [12] used a Moire topography camera to measure the breasts' asymmetry objectively which was followed by a subjective assessment of breast atrophy, skin change and surgical scarring. This introduced the idea of an overall aesthetic assessment, which was expressed by the combination of the individual subjective and objective scores. Glener [13] recently showed that volumetric symmetry is attainable after unilateral autologous breast reconstruction despite the natural fluctuations in the patients' BMI. A symmetry score has been used here where the ratio between the volumes of reconstructed and non-reconstructed breasts at a given time is calculated.

Using a method similar to Noguchi [12], Al-Ghazal [14,15] performed an overall assessment by comparing the subjective evaluation of a six member panel and the patients with the objective scores which were based on the measures of breast retraction, nipple deviation as well as subjective factors such as skin atrophy, skin changes and surgical scars. This method was further developed by Krishnan [16] who added four individual ratings - volume difference, breast asymmetry, fibrosis and telangiectasia-to create an overall cosmetic index which later had a pioneering role in the development of two objective scoring systems using frontal 2D images: the Breast Analyzing Tool (BAT) $[7,8,17,18]$ and the Breast Cancer Conservative Treatment Cosmetic Result (BCCT.core) [4, 19]. In addition to the main three parametersbreast area, breast circumference and nipple position-which are evaluated in the BAT score, BCCT.core also analyzes the color differences and surgical scars along with asymmetry and has been used following a wide variety of procedures including breast conserving surgery [19] and other therapeutic mammoplasty $[20,21]$. Up until this point, most of the development in the objective evaluation of breast asymmetry was based on 2-D images of the patient.

Moyer [22] first introduced an objective technique that proposed a breast asymmetry score based on $3 \mathrm{D}$ surface scans and volume differences using a 3dMD digital camera and 3dMD patient software. Eder et al. [23] superimposed the mirrored breast images to quantify the mean $3 \mathrm{D}$ contour difference by using eight anatomical landmarks which showed better precision than BCCT.core software. Using a portable Artec Eva Scanner, three-dimensional surface images (3D-SI) were recorded for the analysis of breast volumes, and changes in skin appearances for patients receiving radiation treatment [24]. Since both the breast volume and shape symmetry correlated strongly with the subjective assessment scores, the 3D-SI technique was proven to be fast and reliable in determining the breast aesthetic outcome [25].

Further development in the objective assessment of breast asymmetry came to light with the application of convolutional neural network architecture for asymmetry recognition in mammography breast 
imaging data [26]. Another novel visual breast assessment method uses an optical flow algorithm in MATLAB which objectively classifies and numerically scores breast asymmetry [27]. Considering all the statistical data, 3D objective assessment has been proven to be more consistent and accurate, leading it to be a prospective replacement of subjective panel assessment in terms of aesthetic evaluation after breast conserving treatment [28].

In this study, 3D surface scans of sixty patients were taken into account, each of whom was photographed using a Canfield 3D Imager both before and after undergoing various surgical procedures (including BCCT, TRAM flap reconstruction, implant reconstruction or aesthetic alteration). Certain aspects of breast asymmetry parameters were excluded in this score such as: nipple location and distance to the breast border. Future scopes involve the inclusion of breast texture in determining the overall breast symmetry score.

\section{MATERIALS AND METHODS}

\subsection{Subjective Evaluation}

Based on visually detectable differences between the two breasts assessed using 2D and 3D images, the patients were stratified into one of three cohorts by subjective analysis of their breast symmetry: excellentnearly identical breasts, fair-detectable differences between breasts but no serious distortion, poor-detectable asymmetry and distortion of breasts. The images were then further analyzed using the subjective Breast Asymmetry Score (BAS) developed by Bajaj et al. [29] as an additional means of subjectively classifying the patients by symmetry score. The BAS score is determined using a seven-step asymmetry evaluation that grades nipple position, breast shape and ptosis as well as the presence or absence of size asymmetry, skin changes and scarring. The individual breast scores can range from -3 to +6 points, and the final score for each patient is determined by calculating the difference between the two breasts. Scores lie between 0 and 9 with higher scores indicating more asymmetry between the breasts.

\subsection{Objective Evaluation}

The objective symmetry measurements we conducted included the BRA (breast retraction assessment) developed by Pezner [10], the BSI (Breast Symmetry Index) score by Krois et al. [7], the BCCT score by Cardoso et al. [4] and the 3dMD score by Losken et al. [30]. Each of the above scores was calculated for each subject in the study according to the steps outlined in the respective papers.

The BRA is calculated by the formula $\sqrt{\left[\left(\mathrm{X}_{1}-\mathrm{X}_{2}\right)^{2}+\left(\mathrm{Y}_{1}-\mathrm{Y}_{2}\right)^{2}\right]}$ where $X$ is the distance from mid-clavicular line to sternum and $Y$ is the distance from mid-clavicular line to nipple. Although this measure does not afford a global measure of breast similarity, it has been found to correlate fairly well with the degree of asymmetry in a given pair of breasts. The calculation of the BSI score involves the measurement of breast circumference and area along with the distance from the nipple to sternal notch in both the frontal and side planes using 2D images (Figure 1(a) and Figure 1(b)). The difference factor (dF-how many times larger one breast is than the other) and \% difference (\% $\mathrm{d}-$ how much bigger in the amount one breast is than the other) between the right and left breasts (in each of the three planes) are determined and these values are averaged to find the total $\mathrm{dF}$ and \%d between the two breasts. For calculation of the total $\% \mathrm{~d}$, we used a weighted average (weight $=$ area) to give us a more representative indication of symmetry. Figure 1: $\mathrm{c}$ illustrates the symmetry measurements that are performed for the calculation of the BCCT score. These measurements include: BRA measurement, the difference in the breast contours as well as the difference between the levels of the lower breast contours, nipple retraction in the upward direction, differences in breast area and breast overlap along with the evaluation of breast compliancewhich indicates the difference between the left and right nipple-inframammary fold distances-all of which are taken into account in terms of $2 \mathrm{D}$ images. The difference in measurements between the two breasts was converted into a \% difference, representing the symmetry score. The BCCT score also includes color difference and scar visibility, two important but more complicated parameters that we chose not to 


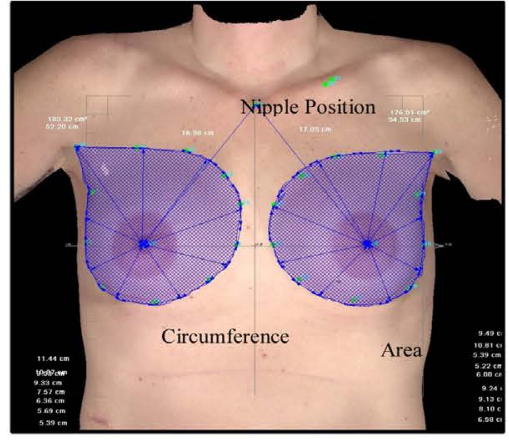

(a)

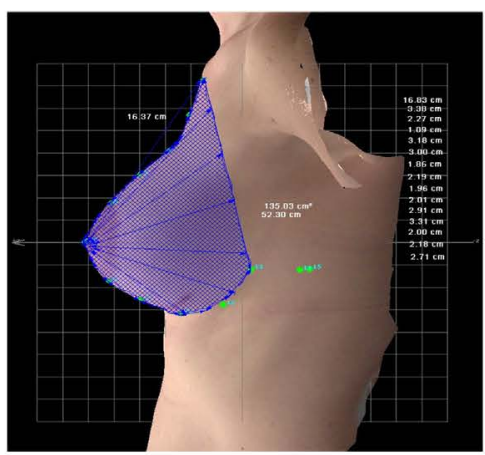

(b)

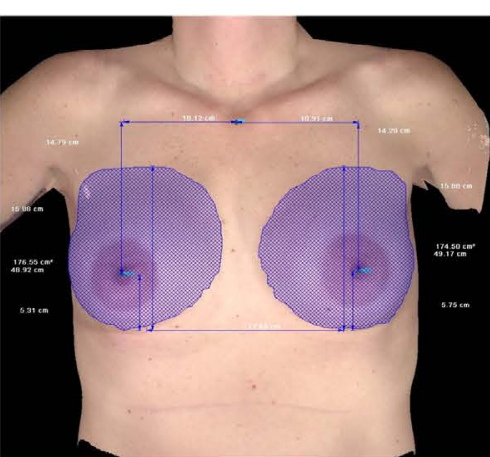

(c)

Figure 1. (a) Front and (b) Lateral 2D Images with BSI Measurements, (c) 2D Image with BCCT Measurements.

include in this particular study. Losken's [30] 3dMD score is calculated by finding the color volume difference between the right and left breasts using 3D images. Using this method, a mirror image of the breast is superimposed over the original breast and the volume difference between the two surfaces is calculated using the Vectra 3D software. The RMS (root mean squared) value is used as the symmetry score (referred as 3D volume diff).

In addition to these three pre-existing objective scores, a new set of measurements was calculated on 3D photographs of the patients, named the BRAS (Breast Retraction Assessment Symmetry) score. This new symmetry score measures the mid-clavicle-sternal notch, mid-clavicle-nipple, sternal notch-nipple, upper breast contour-inframammary fold, lateral mammary fold-medial mammary fold and nipple-inframammary fold distances. The difference in measurements between the two breasts was converted into a \% difference, representing the patient's symmetry score.

These symmetry scores were then compared across individual patients and descriptive statistics were performed to see if the scores were consistent, whether certain parameters were more or less indicative of breast symmetry and whether 3D imaging is necessary to obtain an accurate assessment of breast symmetry. The statistical analysis included calculation of mean, standard deviation, \% differences and Pearson Correlation to show statistically significant differences and correlations. Correlations were plotted as linear regression.

\section{RESULTS AND DISCUSSION}

\subsection{Correlation between Scores}

The BSI score was evaluated for the three cohorts of patients with good, fair and poor symmetry considering the frontal, side and total \% difference (Table 1) and dF (ratio between the breasts) in Figure 2. The correlation between different symmetry scores analyzed is demonstrated in Figure 3. There was a strong positive correlation between all of the objective symmetry scores ( $\mathrm{r}$ values ranging from $0.85-0.95$, $\mathrm{p}<0.001$ ). The intra-patient score correlation was strongest in patients with good symmetry, while patients with poor symmetry had a weaker correlation between scores. Numerous factors contribute to the aesthetic differences between the breasts, which create asymmetry. The weight of these factors determining symmetry differs from one score to another and large differences in these measures will have a greater impact on symmetry scores that put more weight on these parameters. There was a statistically significant correlation amongst the mean symmetry scores for each cohort of patients (Figure 4). The mean \% difference between right and left breast (the different symmetry scores) in the patients with "good" symmetry fell between $2.01 \%-3.59 \%$, a range of $\approx 1.5 \%$ difference points. For the "fair" patient cohort, this score fell between $5.84 \%-6.97 \%$ and for the "poor" symmetry patient cohort, the mean score fell between $16.0 \%$ $27.53 \%$ (Figure 5). There was also a statistically significant correlation between symmetry scores within each patient (Figure 6). 
Table 1. Analysis of BSI scoring considering the front, lateral and total \% difference for the three cohorts (Poor, Fair and Good) of patients.

\begin{tabular}{cccc}
\hline Symmetry Cohort & Frontal (\%) & Lateral (\%) & BSI Score (\%) \\
\hline Poor & 22.55 & 31.02 & 27.11 \\
Fair & 4.41 & 6.63 & 5.84 \\
Good & 1.95 & 3.14 & 2.01 \\
\hline
\end{tabular}

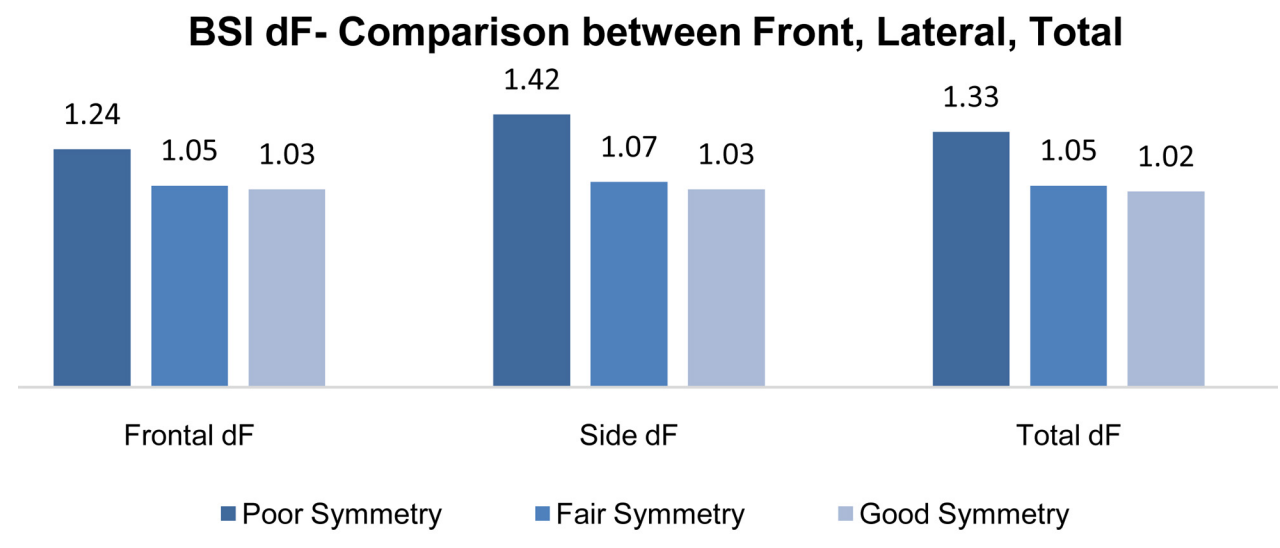

Figure 2. BSI dF scoring for frontal, lateral and total analysis for three cohorts of patients.

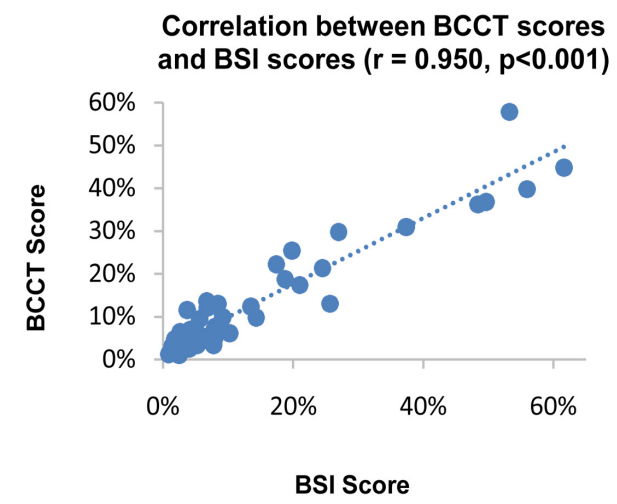

(a)

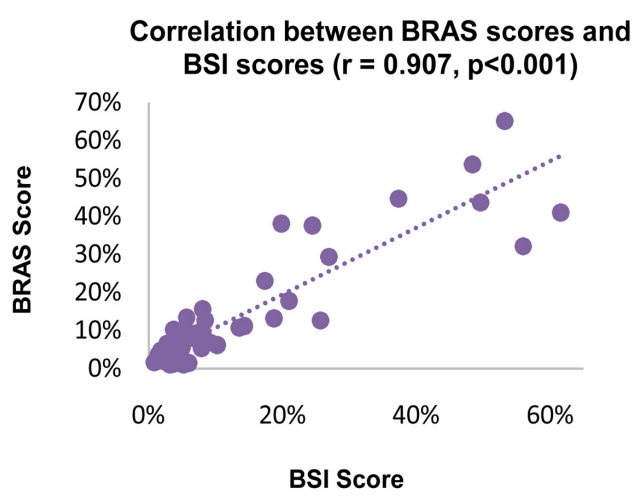

(b)

Figure 3. Pearson correlation between different scores (a) BCCT vs BSI and (b) BRAS vs BSI.

\subsection{Correlation between Objective and Subjective Scores}

There was no strong correlation between the four different objective symmetry scores and the subjective BAS score $(r=0.50)$. However, the mean BAS score for each patient cohort still correlated with the mean objective symmetry scores ( good $=0.45$, fair $=2.8$, poor $=4.05)$.

\subsection{Discussion}

Cosmetic outcome after breast reconstructive surgery has been found to affect the patients' quality of life and psychosomatic wellbeing [14]. As modern treatments have ensured excellent survival rates of 


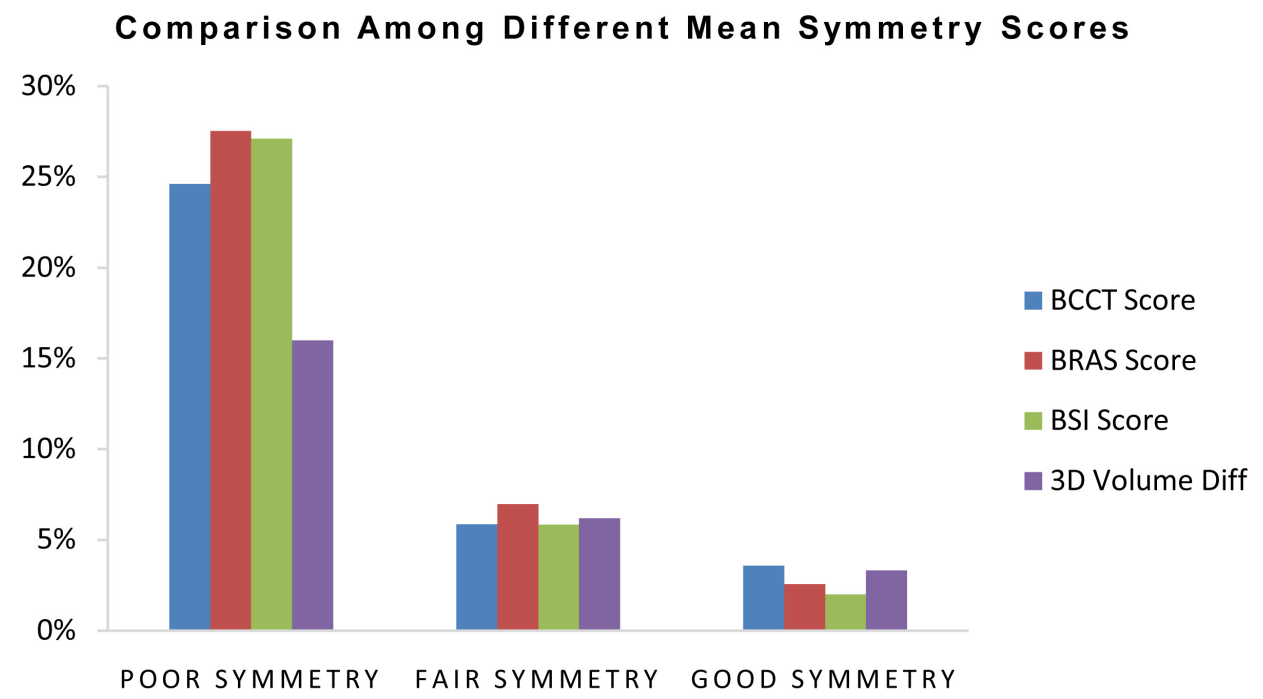

Figure 4. Mean symmetry scores for each patient cohort.

$24.60 \%$

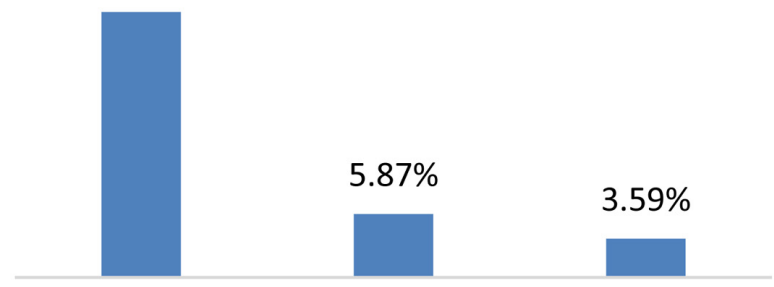

Poor Symmetry Fair Symmetry Good Symmetry

BCCT Score

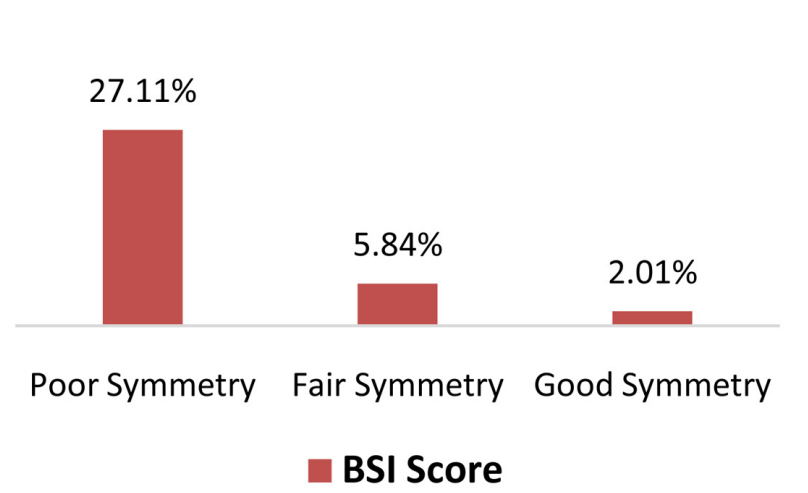

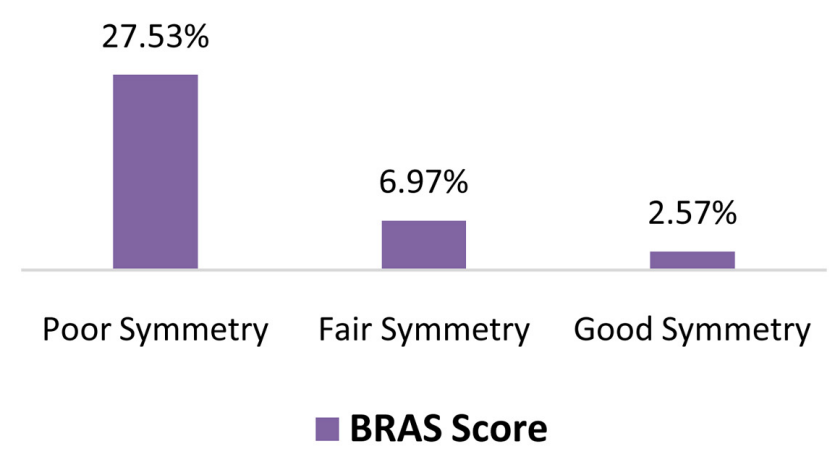

$16.00 \%$

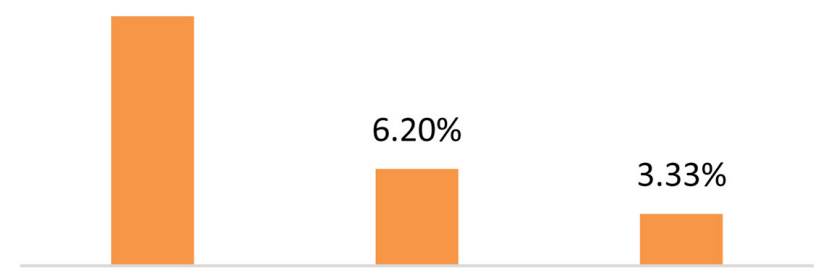

Poor Symmetry Fair Symmetry Good Symmetry

\section{D Volume Diff}

Figure 5. Comparison among different scoring system based on levels of breast symmetry for three cohorts of patients.

breast cancer patients, the aesthetic outcome of the surgical procedure demands more attention in addition to disease control [28]. Subjective evaluation based on a visual assessment by one or more observers, including radiation oncologists and plastic surgeons, are employed frequently. But as the focus is shifting more towards cosmesis, it is crucial to have a reliable, fast and accurate objective assessment criteria for 


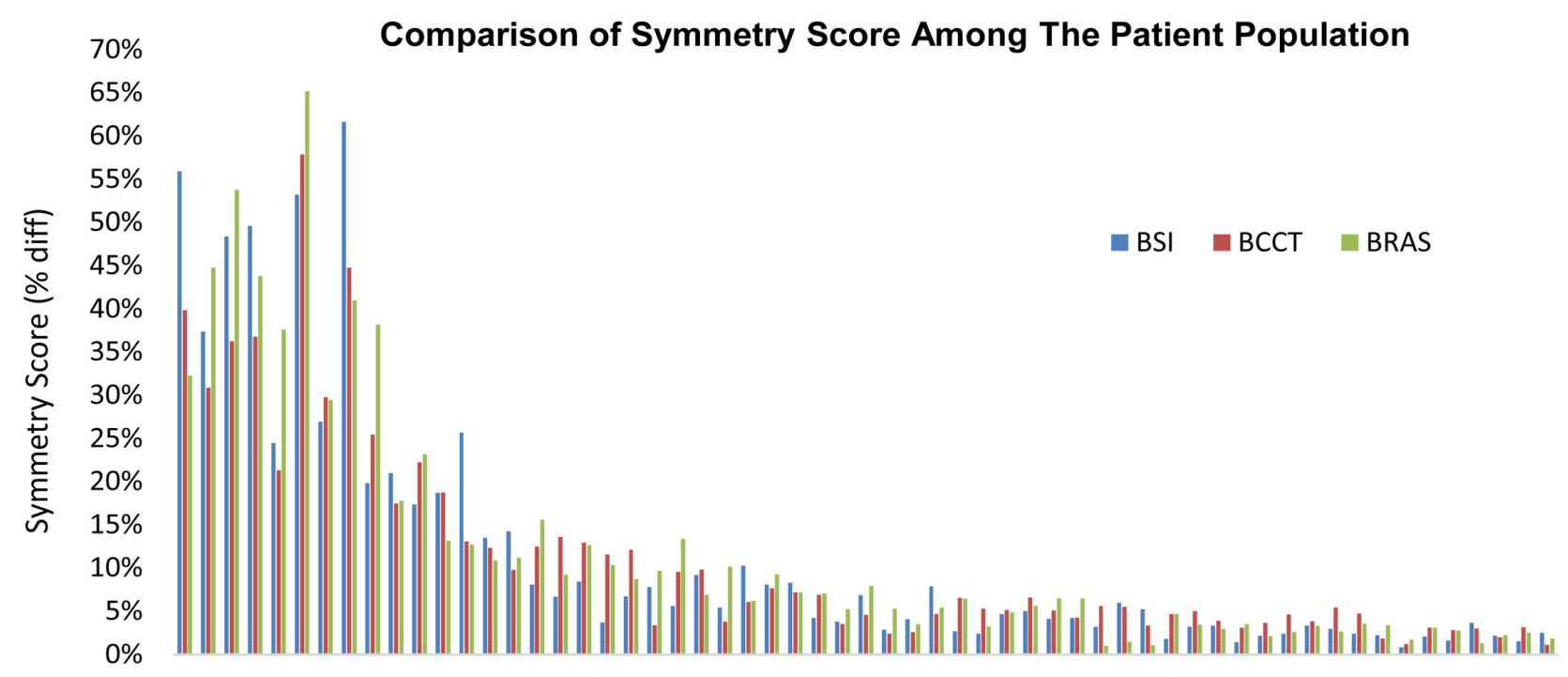

$\begin{array}{llllllllllllllllllllllllllllll}1 & 3 & 5 & 7 & 9 & 11 & 13 & 15 & 17 & 19 & 21 & 23 & 25 & 27 & 29 & 31 & 33 & 35 & 37 & 39 & 41 & 43 & 45 & 47 & 49 & 51 & 53 & 55 & 57 & 59\end{array}$

Number of Patients

Figure 6. Comparison of symmetry score among the patient population.

evaluating breast symmetry that could correlate with the panel assessment scores. Since assessment of breast aesthetics is a complex procedure, a simple, reproducible and ideal breast symmetry assessment technique that is clinically valid is yet to be developed.

For the purpose of this study of determining the advantage of this proposed new score over the preexisting ones, 60 patients were divided into three cohorts: good, fair and poor. Subjective assessment was performed, reaching the conclusion that despite not having a strong correlation, the mean objective scores were comparable to the subjective scores. The objective symmetry scores were studied for each patient and the correlation among the objective scores was consistent for good symmetry.

In this study, we sought to evaluate the existing breast symmetry scores against a new, 3D image-based BRAS score to determine whether there is a need for 3D imaging in the objective evaluation of breast aesthetics. We found that all scores, including our own, correlated for many of the patients in our study. However, for some of the more asymmetrical breasts, where parameters such as nipple position and breast shape were the main factors, several of the preexisting symmetry scores failed to capture the extent of the asymmetry.

Defining the most valid assessment method for cosmetic outcome following breast surgeries still remains challenging due to the need for consideration of different elements. Based on our study, we would propose that certain aesthetic elements dictate the need for 3D imaging to accurately objectify a breast symmetry score.

\section{CONCLUSION}

Over the years, studies have been performed to objectively assess breast aesthetics accurately that can be a reliable alternative to panel assessment. To ensure better scoring, both $2 \mathrm{D}$ and $3 \mathrm{D}$ imaging techniques have been implemented in the objective scoring systems that work with different key measurements. In order to reach the decision regarding the implications of using 3D imaging in objective scoring, further research can be conducted that might include breast texture, biomechanical properties of breast [31] and breast skin [32], scar, and other parameters for a complete breast aesthetic analysis that could eventually replace subjective aesthetic scores especially for post-mastectomy patients.

\section{ACKNOWLEDGEMENTS}

We appreciate the support from Prairie View A\&M University via VPRI-Faculty-RISE. We would like 
to acknowledge Dr. Kathleyn A Brandstetter and Hyo Joon Bang on their primary work in this study. This research was funded in part by the National Science Foundation grant No. 1521801 and also by VeteransAffairs grant 5I01BX000418-06. We also acknowledge the Department of Mechanical and Aerospace Engineering and the Department of Plastic Surgery at the Ohio State University for their financial support during different phases of this work.

\section{CONFLICTS OF INTEREST}

The authors declare no conflicts of interest regarding the publication of this paper.

\section{REFERENCES}

1. Hopwood, P., Fletcher, I., Lee, A. and Al Ghazal, S. (2001) A Body Image Scale for Use with Cancer Patients. European Journal of Cancer, 37, 189-197. https://doi.org/10.1016/S0959-8049(00)00353-1

2. Ng, S.K., Hare, R.M., Kuang, R.J., Smith, K.M., Brown, B.J. and Hunter-Smith, D.J. (2016) Breast Reconstruction Post Mastectomy: Patient Satisfaction and Decision Making. Annals of Plastic Surgery, 76, 640-644.

https://doi.org/10.1097/SAP.0000000000000242

3. Pusic, A.L., Klassen, A.F., Scott, A.M., Klok, J.A., Cordeiro, P.G. and Cano, S.J. (2009) Development of a New Patient-Reported Outcome Measure for Breast Surgery: The BREAST-Q. Plastic and Reconstructive Surgery, 124, 345-353. https://doi.org/10.1097/PRS.0b013e3181aee807

4. Cardoso, M.J., Cardoso, J., Amaral, N., Azevedo, I., Barreau, L., Bernardo, M., Christie, D., Costa, S., Fitzal, F., Fougo, J.L., Johansen, J., Macmillan, D., Mano, M.P., Regolo, L., Rosa, J., Teixeira, L. and Vrieling, C. (2007) Turning Subjective into Objective: The BCCT.core Software for Evaluation of Cosmetic Results in Breast Cancer Conservative Treatment. Breast, 16, 456-461. https://doi.org/10.1016/j.breast.2007.05.002

5. Henseler, H., Smith, J., Bowman, A., Khambay, B.S., Ju, X., Ayoub, A. and Ray, A.K. (2013) Subjective versus Objective Assessment of Breast Reconstruction. Journal of Plastic, Reconstructive \& Aesthetic Surgery, 66, 634-639. https://doi.org/10.1016/j.bjps.2013.01.006

6. Cardoso, M.J., Cardoso, J., Santos, A.C., Barros, H. and de Oliveira, M.C. (2006) Interobserver Agreement and Consensus over the Esthetic Evaluation of Conservative Treatment for Breast Cancer. Breast, 15, 52-57. https://doi.org/10.1016/j.breast.2005.04.013

7. Krois, W., Riedl, O., Dubsky, P., Jakesz, R., Gnant, M., Wild, T. and Fitzal, F. (2009) Objective Cosmetic Analyses after Breast Surgery with the Breast Analysing Tool (BAT) Correlates with Subjective and Objective Scores: A New Tool for Prospective Trials. Cancer Research, 69, 345s-345s.

https://doi.org/10.1158/0008-5472.SABCS-5150

8. Krois, W., Wild, T. and Fitzal, F. (2010) Objective Cosmetic Analysis after Breast Surgery with the Breast Analysing Tool (BAT) Correlates with Subjective Scores: Improved Tool for Clinical Trials. European Journal of Cancer Supplements, 8, 234-235. https://doi.org/10.1016/S1359-6349(10)70624-8

9. Pezner, R.D., Lipsett, J.A., Vora, N.L. and Desai, K.R. (1985) Limited Usefulness of Observer-Based Cosmesis Scales Employed to Evaluate Patients Treated Conservatively for Breast-Cancer. International Journal of Radiation Oncology Biology Physics, 11, 1117-1119. https://doi.org/10.1016/0360-3016(85)90058-6

10. Pezner, R.D., Patterson, M.P., Hill, L.R., Vora, N., Desai, K.R., Archambeau, J.O. and Lipsett, J.A. (1985) Breast retraction Assessment-An Objective Evaluation of Cosmetic Results of Patients Treated Conservatively for Breast-Cancer. International Journal of Radiation Oncology Biology Physics, 11, 575-578. https://doi.org/10.1016/0360-3016(85)90190-7

11. Van Limbergen, E., Van der Schueren, E. and Van Tongelen, K. (1989) Cosmetic Evaluation of Breast Conserving Treatment for Mammary Cancer. 1. Proposal of a Quantitative Scoring System. Radiotherapy and Oncology, 


\section{6, 159-167. https://doi.org/10.1016/0167-8140(89)90016-9}

12. Noguchi, M., Saito, Y., Mizukami, Y., Nonomura, A., Ohta, N., Koyasaki, N., Taniya, T. and Miyazaki, I. (1991) Breast Deformity, Its Correction, and Assessment of Breast Conserving Surgery. Breast Cancer Research and Treatment, 18, 111-118. https://doi.org/10.1007/BF01980973

13. Glener, A.D., Suresh, V., Shammas, R.L., Broadwater, G., Sergesketter, A., Taskindoust, M., Guo, X. and Hollenbeck, S.T. (2019) Volumetric Symmetry after Unilateral Autologous Breast Reconstruction: A Reasonable Goal. Plastic and Reconstructive Surgery-Global Open, 7, e2362. https://doi.org/10.1097/GOX.0000000000002362

14. Al-Ghazal, S.K., Blamey, R.W., Stewart, J. and Morgan, A.A.L. (1999) The Cosmetic Outcome in Early Breast Cancer Treated with Breast Conservation. European Journal of Surgical Oncology, 25, 566-570. https://doi.org/10.1053/ejso.1999.0707

15. Al-Ghazal, S.K., Fallowfield, L. and Blamey, R.W. (1999) Patient Evaluation of Cosmetic Outcome after Conserving Surgery for Treatment of Primary Breast Cancer. European Journal of Surgical Oncology, 25, 344-346. https://doi.org/10.1053/ejso.1999.0654

16. Krishnan, L., Stanton, A.L., Collins, C.A., Liston, V.E. and Jewell, W.R. (2001) Form or Function? Part 2. Objective Cosmetic and Functional Correlates of Quality of Life in Women Treated with Breast-Conserving Surgical Procedures and Radiotherapy. Cancer, 91, 2282-2287. https://doi.org/10.1002/1097-0142(20010615)91:12<2282::AID-CNCR1259>3.0.CO;2-0

17. Cardoso, M.J., Cardoso, J.S., Wild, T., Krois, W. and Fitzal, F. (2009) Comparing Two Objective Methods for the Aesthetic Evaluation of Breast Cancer Conservative Treatment. Breast Cancer Research and Treatment, 116, Article No. 149. https://doi.org/10.1007/s10549-008-0173-4

18. Krois, W., Romar, A.K., Wild, T., Dubsky, P., Exner, R., Panhofer, P., Jakesz, R., Gnant, M. and Fitzal, F. (2017) Objective Breast Symmetry Analysis with the Breast Analyzing Tool (BAT): Improved Tool for Clinical Trials. Breast Cancer Research and Treatment, 164, 421-427. https://doi.org/10.1007/s10549-017-4255-Z

19. Baldacchino, V.R., Bellizzi, A., Madada-Nyakauru, R.N., Kazzazi, F., Oni, G., Forouhi, P. and Malata, C.M. (2019) Assessment of Breast Symmetry in Breast Cancer Patients Undergoing Therapeutic Mammaplasty Using the Breast Cancer Conservation Therapy Cosmetic Results Software (BCCT.core). Gland Surgery, 8, 218-225. https://doi.org/10.21037/gs.2018.09.06

20. Yu, T., Eom, K.-Y., Jang, N.Y., Kim, K.S., Koo, T.R., Kwon, J., Kim, B.H., Kang, E., Kim, S.-W. and Kim, J.-S. (2016) Objective Measurement of Cosmetic Outcomes of Breast Conserving Therapy Using BCCT.core. Cancer Research and Treatment, 48, 491-498. https://doi.org/10.4143/crt.2015.088

21. Hashem, T. and Farahat, A. (2017) Batwing versus Wise Pattern Mammoplasty for Upper Pole Breast Tumours: A Detailed Comparison of Cosmetic Outcome. World Journal of Surgical Oncology, 15, Article No. 60. https://doi.org/10.1186/s12957-017-1124-5

22. Moyer, H.R., Carlson, G.W., Styblo, T.M. and Losken, A. (2008) Three-Dimensional Digital Evaluation of Breast Symmetry after Breast Conservation Therapy. Journal of the American College of Surgeons, 207, 227-232. https://doi.org/10.1016/j.jamcollsurg.2008.02.012

23. Eder, M., Waldenfels, F.V., Swobodnik, A., Klöppel, M., Pape, A.K., Schuster, T., et al. (2012) Objective Breast Symmetry Evaluation Using 3-D Surface Imaging. The Breast, 21, 152-158. https://doi.org/10.1016/j.breast.2011.07.016

24. Koban, K.C., Etzel, L., Li, Z., Pazos, M., Schonecker, S., Belka, C., Giunta, R.E., Schenck, T.L. and Corradini, S. (2020) Three-Dimensional Surface Imaging in Breast Cancer: A New Tool for Clinical Studies? Radiation Oncology, 15, Article No. 52. https://doi.org/10.1186/s13014-020-01499-2

25. O'Connell, R.L., Di Micco, R., Khabra, K., Wolf, L., deSouza, N., Roche, N., Barry, P.A., Kirby, A.M. and Rusby, 
J.E. (2017) The Potential Role of Three-Dimensional Surface Imaging as a Tool to Evaluate Aesthetic Outcome after Breast Conserving Therapy (BCT). Breast Cancer Research and Treatment, 164, 385-393.

https://doi.org/10.1007/s10549-017-4256-y

26. Zeng, Y.-C. (2019) Asymmetry Recognition of Mammogram Images Based on Convolutional Neural Network. 2019 IEEE 8 th Global Conference on Consumer Electronics (GCCE), Osaka, 15-18 October 2019, 738-740. https://doi.org/10.1109/GCCE46687.2019.9015370

27. Monton, J., Kenig, N., Insausti, R. and Jordan, J. (2020) Visual Breast Asymmetry Assessment with Optical-Flow Algorithm. Journal of Medical Systems, 44, Article No. 155. https://doi.org/10.1007/s10916-020-01630-6

28. Godden, A.R., O'Connell, R.L., Barry, P.A., Krupa, K.C.D., Wolf, L.M., Mohammed, K., Kirby, A.M. and Rusby, J.E. (2020) 3-Dimensional Objective Aesthetic Evaluation to Replace Panel Assessment after Breast-Conserving Treatment. Breast Cancer, 27, 1126-1136. https://doi.org/10.1007/s12282-020-01117-9

29. Bajaj, A.K., Kon, P.S., Oberg, K.C. and Miles, D.A. (2004) Aesthetic Outcomes in Patients Undergoing Breast Conservation Therapy for the Treatment of Localized Breast Cancer. Plastic and Reconstructive Surgery, 114, 1442-1449. https://doi.org/10.1097/01.PRS.0000138813.64478.A7

30. Losken, A., Seify, H., Denson, D.D., Paredes Jr., A.A., and Carlson, G.W. (2005) Validating Three-Dimensional Imaging of the Breast. Annals of Plastic Surgery, 54, 471-476; discussion 477-478.

https://doi.org/10.1097/01.sap.0000155278.87790.a1

31. Hojan, K. and Manikowska, F. (2017) Can the Weight of an External Breast Prosthesis Influence Trunk Biomechanics during Functional Movement in Postmastectomy Women? BioMed Research International, 2017, Article ID: 9867694. https://doi.org/10.1155/2017/9867694

32. Sutradhar, A. and Miller, M.J. (2013) In Vivo Measurement of Breast Skin Elasticity and Breast Skin Thickness. Skin Research and Technology, 19, E191-E199. https://doi.org/10.1111/j.1600-0846.2012.00627.x 